\title{
INVESTIGACIONES
}

\section{Andamios de retiro gradual. Parte 1: Visibilización del pensamiento en la construcción de explicaciones científicas escolares*}

\author{
Fading scaffolds. Part 1: Making thinking visible through \\ school scientific explanations \\ Valeria M. Cabello ${ }^{a}$, Martin Sommer Lohrmann ${ }^{b}$ \\ ${ }^{a}$ Pontificia Universidad Católica de Chile, Facultad de Educación. \\ vmcabello@uc.cl \\ ${ }^{b}$ Colegio Alemán de Santiago. \\ masommer@dsstgo.cl
}

\begin{abstract}
RESUMEN
En este artículo se estudia la explicación científica escolar y los andamios de retiro gradual como soporte pedagógico contingente, basado en teorías socioconstructivistas del aprendizaje. Su relevancia se funda en la posibilidad de orientar la práctica en educación en ciencias sobre andamios de retiro gradual, su diseño y aplicación en el soporte del proceso de aprendizaje, específicamente al construir explicaciones científicas escolares por parte de los y las estudiantes. Se discute la relación entre las explicaciones iniciales de los y las estudiantes, las explicaciones que construyen en su escolaridad y las oportunidades de visibilización de su pensamiento que entregan los sistemas de andamiaje, a partir de estudios nacionales e internacionales. Finalmente, se presentan las características fundamentales de los andamios de retiro gradual que ayudan a visibilizar el pensamiento, como propuestas de lineamientos al diseño de soportes pedagógicos basados en investigación y teoría en educación en ciencias.
\end{abstract}

Palabras claves: "andamiaje", "pensamiento científico", "explicación”, “aprendizaje en ciencias".

\begin{abstract}
This article studies the school scientific explanation and scaffolding as contingent pedagogical support, based on socio-constructivist theories of learning. The relevance of this work is guiding science education teaching practice on fading scaffolds, the design and application for supporting the learning process, especially when students construct scientific explanations in the school. The relationship between the students' initial explanations, the explanations they construct in the classroom and the opportunities to make their thinking visible through scaffolding is discussed based on national and international studies. Finally, the fundamental characteristics of scaffolding that help to make thinking visible are presented, as a proposal of guidelines for the design of pedagogical supports derived from science education research and theory.
\end{abstract}

Key words: scaffolding, scientific thinking, explanation, science learning.

\footnotetext{
* Agradecimientos a la Comisión Nacional de Investigación Científica y Tecnológica CONICYT/FONDECYT/11181050, actualmente Agencia Nacional de Investigación y Desarrollo ANID/ FONDECYT/ 11181050.
} 
Estudios Pedagógicos XLVI N 1: 257-267, 2020

ANDAMIOS DE RETIRO GRADUAL. PARTE 1: VISIBILIZACIÓN DEL PENSAMIENTO EN LA CONSTRUCCIÓN DE EXPLICACIONES CIENTÍFICAS ESCOLARES

\section{INTRODUCCIÓN}

La habilidad de construir explicaciones sobre fenómenos naturales se enmarca en el contexto de las habilidades cognitivo-lingüísticas, que se definen como aquellas que se desarrollan para comunicar el conocimiento, ya sea en forma oral o escrita. Explicar fenómenos naturales usando evidencia, teorías o modelos requiere de un razonamiento científico y causal (Legare, Wellman y Gelman, 2009; Tang, 2015, 2016). De hecho, en términos cognitivos, explicar científicamente es una de las operaciones más importantes y complejas (Gilbert, Boutler y Rutherford, 2000; Rappa y Tang, 2018; Thagard, 2012).

Las evaluaciones estandarizadas que incluyen un componente sobre formular explicaciones científicas de fenómenos cotidianos han encontrado que los y las estudiantes presentan dificultades persistentes para explicar. De hecho, en Chile por ejemplo un $70 \%$ de los y las estudiantes en el nivel socioeconómico bajo no es capaz de explicar un fenómeno cotidiano haciendo uso de algún concepto científico (OCDE, 2016). Esto es crítico ya que explicar es una competencia científica y comunicativa que moviliza la comprensión de fenómenos de la naturaleza, al requerir la organización de las variables en un modelo causal (Martín-Díaz, 2013), además de ser clave para entender la naturaleza del conocimiento científico (De Andrade, Freire y Baptista, 2019).

Visualizar la comprensión de los fenómenos a través de la comunicación se sostiene a partir de la configuración de las mediaciones entre los esquemas cognitivos del aprendiz y el contexto de referencia, en el caso de la escolaridad, contexto que viene dado por el(la) docente o los recursos pedagógicos que se dispongan para posibilitar el aprendizaje (De Longhi et al., 2012). El mismo autor enfatiza la estrecha ligazón entre el lenguaje y la ciencia, ya que aprender ciencia incluye apropiarse de los recursos discursivos, de la manera de hablar en esa disciplina, de argumentar, de debatir y de legitimar los conocimientos. En otras palabras, se facilita el aprender ciencias a partir del hablar, leer y escribir ciencia (Sanmartí, 2007; Martín-Díaz, 2013). Desde esta perspectiva, crear oportunidades para los y las estudiantes de verbalizar sus razonamientos, haciendo uso de las habilidades o prácticas lingüísticas es crucial para apoyarlos en dar sentido a las ideas científicas, favorecer la comprensión de los fenómenos y que su pensamiento se haga visible y posible de ser apoyado pedagógicamente (Braaten y Windschitl, 2011; Zangori y Forbes, 2013). De hecho, construir explicaciones permite organizar y unificar el conocimiento, así como avanzar en la comprensión de los fenómenos (Martín Díaz, 2013). Por último, apoyar a los y las estudiantes en su construcción de explicaciones es crucial en el desarrollo de prácticas científicas, ya que la explicación influencia fuertemente otras prácticas claves como la experimentación, la evaluación crítica de evidencia, la contrastación de hipótesis, entre otras (Zimmerman, 2000). Explicar también permite a los y las escolares tomar una posición crítica e informada sobre fenómenos naturales y del entorno que afectan su vida (Martín-Díaz, 2013).

Ante la necesidad de apoyar a los y las estudiantes en el proceso de visibilizar la comprensión de los fenómenos a través de explicaciones (De Andrade, Freire y Baptista, 2019, Zangori y Forbes, 2013), ofrecer una estructura determinada -como un andamio de retiro gradual que se revisará en este estudio- es un apoyo al desarrollo de sus explicaciones (Park, Chang, Tang, Treagust \& Won, 2020), y a distinguir entre descripciones y explicaciones (Braaten y Windschitl, 2011; Zangori y Forbes, 2013). Lo anterior, ya que explicar es una habilidad distinta que describir o justificar (Jorba, Gómez y Prat, 2000), idea que se profundizará en el siguiente apartado. 
Considerando lo anterior, la pregunta que abordamos en este trabajo es: ¿Cuáles son los sustentos empíricos y teóricos asociados al desarrollo de explicaciones científicas escolares mediante andamios de retiro gradual?

A continuación, se aborda la discusión sobre el concepto de explicación científica escolar en educación en ciencias, y se propone una conceptualización para este estudio que discute los apoyos pedagógicos a su construcción, a partir de la noción de andamiajes de retiro gradual. Se toma posicionamiento en relación con la noción de sistemas de andamios para visibilizar el pensamiento y apoyar el aprendizaje, a partir de estudios nacionales e internacionales que han aplicado andamiajes en la enseñanza de las ciencias naturales. Finalmente, se discuten las características centrales de los andamios de retiro gradual con la finalidad de potenciar el desarrollo de explicaciones científicas escolares por parte de estudiantes, como una propuesta que avance en los lineamientos al diseño de soportes pedagógicos estratégicamente implementados, basados en investigación y teoría para la educación en ciencias.

\section{MARCO TEÓRICO}

Explicar es una habilidad cognitivo-lingüística, capaz de progresar durante la escolaridad (Gotwals y Songer, 2013; Yao y Guo, 2018). Cognitiva, en la medida que estructura el razonamiento mediante relaciones causales entre hechos, fenómenos y conceptos, lingüística, pues toma una forma discursiva oral o escrita con estructura textual causal (Jorba, Gómez y Prat, 2000). El lenguaje tiene un papel preponderante en la construcción de explicaciones (Rappa y Tang, 2018), y en el aprendizaje, ya que representa el saber y, a la vez, media su construcción (Legare, Wellman y Gelman, 2009; Meneses, Hugo, Montenegro, Valenzuela y Ruiz, 2018). De hecho, la capacidad de aprovechar las explicaciones del estudiantado sobre los fenómenos naturales como hipótesis que pueden ser desafiadas, complementadas y sometidas a verificación es uno de los estándares orientadores de la formación docente en Chile dada su relevancia en el aprendizaje de las ciencias (MINEDUC, 2011).

\subsection{EXPLICACIONES INICIALES Y EXPLICACIÓN CIENTÍFICA ESCOLAR}

Las ideas o explicaciones iniciales de los y las estudiantes en ciencias se han conceptualizado de diversas maneras, entremezclando con nociones como los preconceptos, modelos explicativos, concepciones alternativas o ideas intuitivas (Barke, Hazari y Yitbarek, 2009; Benarroch, 2001; Carrascosa, 2005; Concari, 2001; Driver, Newton y Osborne, 2000). Una posición posible en esta polisemia del constructo es entender que las "explicaciones iniciales" de los y las estudiantes son aquellas previas a la instrucción formal, mientras que el marco explicativo o explicación científica escolar se construyen mediadas por una enseñanza explícita e intencionada (Eberbach y Crowley, 2009; Márquez, Roca, Gómez, Sardá y Pujol, 2004). Las explicaciones científicas escolares son entendidas en este estudio como una construcción lingüística y cognitiva que hace una aplicación explícita de alguna teoría, que trasciende la descripción de patrones, para revelar las relaciones causales o mecanismos subyacentes a una situación o fenómeno (Yao y Guo, 2018). En la clase de ciencias, la explicación científica escolar apunta a que los y las estudiantes demuestren comprensión de los fenómenos naturales y no se refiere aclaraciones de conceptos o 
razonamientos aplicados a la resolución de problemas, empleados frecuentemente en clases de ciencias (Braaten y Windschitl, 2011). Los mismos autores indican que las explicaciones científicas escolares requieren, por tanto, de la sustentación teórica de las afirmaciones, aludiendo a entidades no visibles a simple vista, teorías o a grandes ideas científicas vinculadas con las causas del fenómeno. Estas entidades no visibles son, para Ogborn, Kress y Martins (1996) uno de los elementos clave que permiten diferenciar una descripción del fenómeno del nivel siguiente, en orden cognitivo, que es explicar la dinámica del fenómeno a partir de aquello invisible a los sentidos, es decir, el por qué subyacente al devenir del fenómeno).

En términos de investigación, en lenguaje español los estudios que abordan esta temática son insuficientes (Meneses et al., 2018). Los estudios en Chile sobre explicaciones científicas escolares han encontrado que los y las estudiantes de educación básica escasamente vinculan los fenómenos con principios subyacentes o evidencia científica en sus explicaciones iniciales (Cabello, 2016; Sommer y Cabello, 2018), lo que coincide con estudios internacionales en niveles similares de escolaridad (Forbes et al., 2014; Songer y Gotwals, 2012). Sin embargo, las explicaciones científicas escolares son susceptibles de ser enriquecidas en unidades pedagógicas intencionadas explícitamente hacia el lenguaje académico (Meneses et al., 2018). En estudiantes de enseñanza media, Moreira, Marzabal y Talanquer (2019), caracterizaron las explicaciones escolares en un tópico de química visibilizando el razonamiento que subyace a las producciones escritas, y, a su vez, describiendo un amplio rango de patrones de pensamiento de los y las estudiantes, el cual en varios casos difería del modelo científico escolar esperado por sus docentes. Consideramos que las explicaciones científicas escolares contribuirían a visibilizar el pensamiento de los y las estudiantes sobre diversos fenómenos -tanto aquellas explicaciones que son iniciales, es decir, previas a la instrucción formal como aquellas que se construyen en la escolaridad-, en especial cuando se utilizan estructuras de apoyo que contribuyen a tomar conciencia sobre las ideas y su estructuración en una producción textual disciplinar específica como lo es la explicación científica escolar.

En educación en ciencias las habilidades cognitivo-lingüísticas más importantes son la descripción, la explicación y argumentación (Jorba et al., 2000; Milne, 2012), las que se influyen mutuamente (Osborne y Patterson, 2011). La validez de una explicación usualmente requiere argumentación y el proceso de argumentación usualmente involucra múltiples explicaciones (Tang, 2016). La explicación busca generar entendimiento sobre un fenómeno basándose en conocimiento científico previo, formular nuevas teorías para comprender las causas o génesis de un fenómeno, mientras que la argumentación busca justificar un hallazgo del conocimiento, persuadir a alguien sobre éste o expandir una controversia a partir, por ejemplo, de un debate (Osborne y Patterson, 2011). La descripción delimita el fenómeno a partir de sus características perceptibles (Zangori y Forbes, 2013), por lo que la explicación supera en complejidad a la descripción y la argumentación implica un nivel distinto de organización y finalidad que la explicación. Al desglosar una explicación en una afirmación y en su fundamento científico, tal como lo proponen Braaten y Windschitl (2011), la tarea de relacionar la afirmación con las causas del fenómeno y con conceptos o teorías científicas se condice más con el concepto de explicación para representar(se) un fenómeno natural que con las habilidades necesarias para producir una explicación en el marco de la argumentación científica. Siguiendo esta línea conceptual, la explicación no se relacionaría directamente con el debate de ideas como la argumentación, sino más bien con la creación de 
sentido o construcción de conocimiento a partir del fenómeno, por ende, es relevante hacer la distinción a la hora de diseñar apoyos pedagógicos que permitan a los y las estudiantes desarrollar cada una de estas habilidades en su especificidad (Berland y McNeill, 2012).

\subsection{SISTEMAS DE ANDAMIOS PARA APOYAR EL APRENDIZAJE}

Los andamios son apoyos que se van adaptando a los avances del proceso de aprendizaje de los y las estudiantes, destinados a satisfacer el objetivo de aprendizaje y pueden ayudarle en la conquista de su zona de desarrollo próximo (en adelante, ZDP) (McNeill, Lizotte, Krajcik y Marx, 2006). Este concepto es entendido como la distancia que existe entre el desarrollo cognitivo de la o del estudiante y su desarrollo potencial, zona en la que se favorece el aprendizaje mediante interacciones intencionadas con algún adulto o par más experimentado en la tarea específica (Cole, 1984; Vigotsky, 1979). Es justamente en esta zona donde los apoyos pedagógicos, entendidos en el marco vigotskiano como andamios (Guilar, 2009), pueden ser incluidos y luego retirados en el momento preciso en que el estudiante es capaz de desarrollar por si solo la tarea, práctica o actividad, con el fin de que el mismo desafío propuesto por el apoyo sea el que movilice el desarrollo de las habilidades que están en juego (Vigotsky, 1979).

Profundizando en los andamios van de Pol, Volman y Beishuizen (2010), en un estudio que recopila una década de investigación sobre andamiajes, argumentan que el concepto de andamio ha sido desvirtuado de su sentido original, confundiéndose muchas veces con recursos pedagógicos comunes que se mantienen sin variación aún si el estudiante ha avanzado en su aprendizaje. Estos autores sostienen que para que un andamio califique como tal debe cumplir con las siguientes características:

1. Contingencia, es decir, incluye y/o permite una constante adaptación a los avances de cada estudiante.

2. Retiro gradual, es decir, posibilidad de retiro adaptativo de estructuras en la medida que el estudiante vaya avanzando en su proceso de aprendizaje. Esto incluye la posibilidad de un retiro total, si la estructura del apoyo ha sido internalizada por el estudiante.

3. Transferencia de responsabilidad, el dispositivo incluye gradualmente la toma de responsabilidad por parte del estudiante en relación con su proceso de aprendizaje. Es decir, mientras menos apoyo requiera el estudiante, mayor será la autonomía que se persiga, por ende, la autonomía que se quiere desarrollar en el estudiante se transfiere paulatinamente a través del retiro gradual de los andamios, proceso en el cual él puede decidir.

Los mismos autores reconocen que estas tres características, sobre todo, la contingencia, forman parte de una enseñanza personalizada, que puede ser difícil de implementar con grupos grandes de estudiantes especialmente para el desarrollo de habilidades de alto orden o complejas.

Construir explicaciones de fenómenos naturales con sustento científico es difícil para los y las estudiantes (Lee y Songer, 2004; McNeill et al., 2006; McNeill y Krajcik, 2009; Ruiz-Primo, Li, Tsai \& Schneider, 2010; Zangori y Forbes, 2013). Que los y las docentes diseñen apoyos específicos a la construcción de explicaciones es una necesidad 
crítica, lo cual favorecería intervenciones pedagógicas específicas según las características de sus estudiantes (Braaten y Windschitl, 2011; Zangori y Forbes, 2013) y la naturaleza de la construcción de explicaciones científicas escolares (Meneses et al., 2018). De no ofrecerse dichos apoyos pedagógicos en la sala de clases, las tareas inherentes al desarrollo de habilidades científicas de complejidad creciente tales como describir, explicar y argumentar, podrían resultar alejadas de la ZDP de los y las estudiantes (Lee y Songer, 2004; McNeill et al., 2006).

Las investigaciones orientadas a apoyar pedagógicamente a los y las estudiantes con sistemas de andamios, ya sea con el fin de desarrollar sus conocimientos científicos y/o sus habilidades o prácticas científicas han ido en aumento internacionalmente en los últimos años (i.e Oktavianti, Handayanto, Wartono y Saniso, 2018; Tang, 2015, 2016). De aquellas investigaciones que sustentan sus conclusiones en evidencia, se han seleccionado elementos que vislumbran avances respecto a su efectividad, útiles para el diseño e implementación de sistemas de andamiaje, descritos a continuación.

Los andamios escritos han probado ser eficaces para el trabajo con grupos grandes de estudiantes. Aplicando diversas mediciones de la calidad de las explicaciones de estudiantes antes y después de un sistema secuenciado de andamios escritos de retiro gradual, se ha observado que existen avances tanto en la habilidad para explicar, como en la comprensión de los fenómenos naturales (Hsu, Lai y Hsu, 2015; Lee y Songer, 2004; McNeill y Krajcik, 2009; McNeill et al., 2006). En el trabajo en aula con grupos de entre 25 a 45 estudiantes, considerados en este estudio como grupos grandes, los andamios escritos entregados individualmente para que ellos y ellas escriban sus explicaciones, posibilitan al docente entregar una retroalimentación personalizada al revisar sus productos e ir ajustando la estructura de apoyo según el avance personal (Sommer y Cabello, 2018). Por ejemplo, a partir de los avances observados, se pueden volver a entregar los mismos apoyos escritos a quienes los requieran y apoyos reducidos a aquellos(as) estudiantes más avanzados(as).

También se ha determinado que el uso de andamiajes retirados gradualmente mientras los escolares internalizan la estructura explicativa, enriquece sus explicaciones (Sommer y Cabello, 2018), mientras que si se disminuye gradualmente el andamiaje mejora la habilidad de explicar solo si a la vez los niños logran una comprensión conceptual de los principios, ideas o teorías subyacentes al fenómeno (McNeill et al., 2006).

Sin embargo, proveer a los y las estudiantes solamente de un tipo de andamios no constituye necesariamente un apoyo eficaz (Hsu et al., 2015; McNeill y Krajcik, 2009). Es decir, la implementación de sistemas de andamiajes amplios y variados, llamados "distributivos" -por ejemplo, el apoyo directo del (de la) docente, trabajo entre pares o grupos pequeños, ejercicios metacognitivos, entre otros-, son positivos para generar una sinergia que contribuya en su conjunto a apoyar al estudiantado en sus progresos en la habilidad de explicar (Hsu et al., 2015; Park et al., 2020; Rappa y Tang, 2018). La condición de contingencia, es decir, la adaptación a los avances de cada individuo se potencia mediante los andamios distributivos al compensar las limitaciones de los andamios escritos cuando son aplicados como única alternativa (Hsu et al., 2015).

Dado lo anterior, un sistema de andamios adecuado para el desarrollo de explicaciones debiera incluir tanto andamios genéricos, es decir, que apoyen el desarrollo de la habilidad para explicar fenómenos, como andamios de contenidos, es decir, aquellos orientados a apoyar el aprendizaje de conceptos, teorías o postulados científicos involucrados y su aplicación a los fenómenos (McNeill y Krajcik, 2009). De hecho, estos mismos autores 
observaron avances en la explicación de fenómenos al realizar cambios de temática a explicar, gracias a un mayor apoyo mediante andamios de contenidos.

Adicionalmente, se ha planteado la utilidad de implementar sistemas de andamiajes enmarcados en los métodos de enseñanza que integren el ciclo indagatorio, ya sea, mediante el aprendizaje de las ciencias basado en indagación, en la resolución de problemas, en proyectos u otros (Hsu et al., 2015). Este ciclo se inserta en un enfoque metodológico que insta a los y las estudiantes a generar explicaciones como respuestas a problemas o preguntas (Hsu et al., 2015), haciendo uso de las formas en las cuales los científicos construyen conocimiento y proponen explicaciones (Garritz, 2010). Si bien el proceso indagatorio no necesariamente implica una secuencia lineal de pasos (Newman et al., 2004), incluye elementos relacionados con las habilidades científicas de identificación y focalización en un problema o pregunta, exploración de posibles respuestas o antecedentes, formulación de hipótesis, modelos y/o explicaciones junto a su puesta a prueba, y la aplicación de los nuevos conocimientos en función del problema o pregunta (Abd-ElKhalick et al., 2004). Estos procesos permiten ganar creciente autonomía en el estudiantado cuando da sustento a sus explicaciones, visibilizando su pensamiento y aproximándose sucesivamente a las prácticas científicas (Hsu et al., 2015; McNeill y Krajcik, 2009; McNeill et al., 2006).

A pesar de la efectividad demostrada en algunos estudios internacionales (Hsu et al., 2015; Lee y Songer, 2004; McNeill y Krajcik, 2009; McNeill et al., 2006), un desarrollo de sistemas de andamiaje diverso y adaptable a los avances de los y las estudiantes sin una adecuada preparación docente no es garantía de un proceso exitoso para desarrollar explicaciones científicas escolares. Una investigación realizada por Zangori y Forbes (2013) con futuros maestros y sus estudiantes concluyó que dependía de la preparación de las y los futuros profesores(as) el apoyo que podían proveer a sus estudiantes en la formulación de explicaciones. Observaron también que si las nociones acerca de lo que es -y no es- una explicación científica no estaba clara para el profesorado en formación y si, a su vez, las prácticas para apoyar a los y las estudiantes eran inadecuadas, sus intervenciones en clases de ciencias no fomentaban adecuadamente el desarrollo de explicaciones de fenómenos naturales por parte de los y las estudiantes.

\section{DISCUSIÓN Y CONSIDERACIONES PARA LA PRÁCTICA}

Aprender ciencias implica adquirir un nuevo vocabulario, una nueva semántica y construcción de significados -oraciones, párrafos- en donde se usen los términos relacionados en variados contextos (Lemke, 1990). Aunque los y las estudiantes sepan palabras con significado científico, algunos autores han indicado que cuando explican -a sí mismos o a otros- se pone realmente en juego su comprensión conceptual (LeymoniéSáenz, 2009; Lee y Songer, 2004; Martín-Díaz, 2013; Sanmartí, 2007).

A mayor complejidad del fenómeno a explicar, mayor necesidad de un lenguaje que permita representar, organizar y comunicar esa experiencia y sus relaciones (Izquierdo y Aliberas, 2004). Esto permitiría proyectar una hipótesis de progreso, entendida como la naturaleza y la secuencia de las habilidades y de contenidos que los y las estudiantes deberían desarrollar a lo largo de una secuencia curricular (Songer, Kelcey y Gotwals, 2009; Songer y Gotwals, 2012; Yao y Guo, 2018). 
Respecto de la pregunta que se planteó abordar este artículo, se identificaron estudios que en sus resultados evidenciaron progresos en la construcción de explicaciones de los y las estudiantes. A continuación, se sintetizan a modo de recomendaciones para tener en cuenta para el diseño en el aula:

a) La continua evaluación del proceso de aprendizaje, que incluye las explicaciones iniciales y permite responder a la contingencia de los avances particulares de los y las estudiantes (Sommer y Cabello, 2018; Yao y Guo, 2018).

b) Las estrategias didácticas que integran el ciclo indagatorio dan un sustento empírico y conceptual que permite fundamentar y poner a prueba las explicaciones escolares iniciales (Hsu et al., 2015).

c) Los andamios escritos permiten trabajar en el aula de clases con grandes grupos, manteniendo sus características si se cuida la individualización y contingencia (Hsu et al., 2015; Lee y Songer, 2004; McNeill y Krajcik, 2009; McNeill et al., 2006).

d) La distinción y articulación de andamios genéricos y de contenidos permiten apoyar tanto la construcción de explicaciones como el desarrollo comprensivo de conceptos, teorías o postulados científicos relacionados con los fenómenos a explicar (McNeill y Krajcik, 2009).

e) Los andamios distributivos apoyan a los andamios escritos generando sinergia de apoyos que pueden potenciar el aprendizaje, si son aplicados estratégica y flexiblemente (Hsu et al., 2015).

La progresión de la habilidad de explicar, en educación en ciencias se puede apoyar a través del diseño de apoyos pedagógicos flexibles y contingentes, que son llamados andamios de retiro gradual en este estudio. Dicha progresión da un marco de coherencia e indica un ancla de menor nivel cognitivo, niveles intermedios y un ancla superior para las explicaciones (Songer y Gotwals, 2012; Yao y Guo, 2018). Según lo revisado en el presente estudio, se puede interpretar que el nivel inferior correspondería a las explicaciones iniciales del estudiantado, influidas por múltiples fuentes y los niveles sucesivos a las explicaciones científicas escolares en sus diversos grados de complejización, para llegar a un nivel más alto acercándose al modelo científico actual. Esto les permitiría la generación de múltiples explicaciones para fenómenos diferentes a aquellos en los que se originaron (Lehrer y Schauble, 2006; Schwarz et al., 2009). Lo anterior podría formar parte de las proyecciones de este estudio para generar nuevas preguntas.

La implementación de una unidad pedagógica sobre la base de los postulados anteriores, así como los instrumentos de evaluación formativa que contribuyen a visibilizar el pensamiento de los y las estudiantes, ejemplos de actividades y resultados obtenidos se presenta en la segunda parte de este trabajo, titulado "Andamios de retiro gradual. Parte 2: Apoyos a la construcción de explicaciones en ciencia primaria".

\section{REFERENCIAS BIBLIOGRÁFICAS}

Abd-El-Khalick, F., Boujaoude, S., Duschl, R., Lederman, N. G., Mamlok-Naaman, R., Hofstein, A., ... \& Tuan, H. L. (2004). Inquiry in science education: International perspectives. Science Education, 88(3), 397-419. Doi: 10.1002/sce.10118 
Braaten, M. y Windschitl, M. (2011). Working toward a stronger conceptualization of scientific explanation for science education. Science Education, 95(4), 639-669. Doi: 10.1002/sce.20449

Barke, H. D., Hazari, A., \& Yitbarek, S. (2009). Students' misconceptions and how to overcome them. In Misconceptions in Chemistry (pp. 21-36). Springer, Berlin, Heidelberg.

Benarroch, A. (2001). Una interpretación del desarrollo cognoscitivo de los alumnos en el área de la naturaleza corpuscular de la materia. Enseñanza de las Ciencias, 19(1), 123-134.

Berland, L. K. y McNeill, K. L. (2012). For whom is argument and explanation a necessary distinction? A response to Osborne and Patterson. Science Education, 96(5), 808-813. Doi: $10.1002 /$ sce. 21000

Cabello, V., (2016). Explanation construction of scientific phenomena: A study of teachers and their students. In J. Lavonen, K. Juuti, J. Lampiselkä, A. Uitto \& K. Hahl (Eds.), Electronic Proceedings of the ESERA 2015 Conference. Science education research: Engaging learners for a sustainable future, Part 16 (co-ed. Petros Kariotoglou \& Terry Russell), (pp. 2760-2769). Helsinki, Finland: University of Helsinki. ISBN 978-951-51-1541-6

Carrascosa, J. (2005). El problema de las concepciones alternativas en la actualidad (parte I). Análisis sobre las causas que la originan y/o mantienen. Revista Eureka sobre Enseñanza y Divulgación de las Ciencias, 2(2),183-208.

Cole, M. (1984). La zona de desarrollo próximo: donde cultura y conocimiento se generan mutuamente. Infancia y Aprendizaje, 7(25), 3-17. Doi: 10.1080/02103702.1984.10822018

Concari, S. B. (2001). Las teorías y modelos en la explicación científica: implicancias para la enseñanza de las ciencias. Ciência \& Educação, 7(1), 85-94.

De Andrade, V., Freire, S., \& Baptista, M. (2019). Constructing scientific explanations: a system of analysis for students' explanations. Research in Science Education, 49(3), 787-807. Doi: 10.1007/s11165-017-9648-9

De Longhi, A. L., Ferreyra, A., Peme, C., Bermudez, G. M. A., Quse, L., Martínez, S., Iturralde, C. \& Campaner, G. (2012). La interacción comunicativa en clases de ciencias naturales. Un análisis didáctico a través de circuitos discursivos. Revista Eureka sobre Enseñanza y Divulgación de las Ciencias, 9(2), 178-195.

Driver, R., Newton, P., \& Osborne, J. (2000). Establishing the norms of scientific argumentation in classrooms. Science Education, 84(3), 287-312.

Eberbach, C., \& Crowley, K. (2009). From everyday to scientific observation: How children learn to observe the biologist's world. Review of Educational Research, 79(1), 39-68.

Forbes, C., Lange, K., Möller, K., Biggers, M., Laux, M., \& Zangori, L. (2014). ExplanationConstruction in Fourth-Grade Classrooms in Germany and the USA: A cross- national comparative video study. International Journal of Science Education, 36(14), 2367-2390. doi: 10.1080/09500693.2014.923950

Garritz, A. (2010). Indagación: las habilidades para desarrollarla y promover el aprendizaje. Educación Química, 21(2), 106-110. Doi: 10.1016/S0187-893X(18)30159-9

Gilbert, J. K., Boulter, C. J., \& Rutherford, M. (2000). Explanations with models in science education. In Developing models in science education (pp. 193-208). Springer, Dordrecht.

Gotwals, A. W., \& Songer, N. B. (2013). Using assessments to gather validity evidence for a learning progression on evidence-based explanations with core ecological content. The Journal of Research in Science Teaching, 40(5), 597-626.

Guilar, E. (2009). Las ideas de Bruner: "de la revolución cognitiva" a la "revolución cultural". Educere, 13(44), 235-241.

Hsu, Y.-S., Lai, T.-L. y Hsu, W.-H. (2015). A design model of distributed scaffolding for inquirybased learning. Research in Science Education, 45(2), 241-273.

Izquierdo, M. y Aliberas, Y. J. (2004). Pensar, actuar i parlar a la classe de ciències. Barcelona: Universitat Autónoma de Barcelona.

Jorba, J., Gómez I., y Prat, A. (2000). Hablar y escribir para aprender. Madrid: Síntesis. 
Estudios Pedagógicos XLVI N ${ }^{\circ} 1: 257-267,2020$

ANDAMIOS DE RETIRO GRADUAL. PARTE 1: VISIBILIZACIÓN DEL PENSAMIENTO EN LA CONSTRUCCIÓN DE EXPLICACIONES CIENTIIFICAS ESCOLARES

Lee, H.-S. \& Songer, N. B. (2004, April). Longitudinal knowledge development: Scaffolds for Inquiry. Paper presented at the annual meeting of the American Educational Research Association, San Diego, CA.

Legare, C. H., Wellman, H. M., \& Gelman, S. A. (2009). Evidence for an explanation advantage in naïve biological reasoning. Cognitive Psychology, 58(2), 177-194.

Lehrer, R. \& Schauble, L. (2006). Cultivating Model-Based Reasoning in Science Education. Cambridge: Cambridge University Press.

Lemke, J. L. (1990). Talking science: Language, learning, and values. New Jersey: Ablex Publishing Corporation.

Leymonié-Sáenz, J. (2009). Aportes para la enseñanza de las ciencias naturales. Santiago de Chile: UNESCO.

Márquez, C., Roca, M., Gómez, A., Sardá, A., y Pujol, R. M. (2004). La construcción de modelos explicativos complejos mediante preguntas mediadoras. Investigación en la Escuela, 53, 71-81.

Martín-Díaz, M. J. (2013). Hablar ciencia: si no lo puedo explicar, no lo entiendo. Revista Eureka sobre Enseñanza y Divulgación de las Ciencias, 10(3), 291-306. Recuperado desde: https:// revistas.uca.es/index.php/eureka/article/view/2843/249

McNeill, K. L., Lizotte, D. J., Krajcik, J. y Marx, R. W. (2006). Supporting Students construction of scientific explanations by fading scaffolds in instructional materials. The Journal of the Learning Sciences, 15(2), 153-191. Doi: 10.1207/s15327809j1s1502_1

McNeill, K. L. y Krajcik, J. (2009). Synergy between teacher practices and curricular scaffolds to support students in using domain-specific and domain-general knowledge in writing arguments to explain phenomena. The Journal of the Learning Sciences, 18(3), 416-460. https://doi. org/10.1080/10508400903013488

Meneses, A., Hugo, E., Montenegro, M., Valenzuela y Ruiz, M. (2018). Explicaciones científicas: propuestas para la enseñanza del lenguaje académico. Boletín de Lingüística, 30(49), 134-157.

MINEDUC, Ministerio de Educación (2011). Estándares orientadores para egresados de pedagogía en educación básica. Recuperado desde https://www.cpeip.cl/wp-content/uploads/2016/07/ librobasicaokdos.pdf

Milne, C. (2012). Beyond argument in science: Science education as connected and separate knowing. In: B. Fraser, K. Tobin \& C. McRobbie (Eds.), Second international handbook of science education. (pp. 951-967). Dordrecht: Springer. Doi: 10.1007/978-1-4020-9041-7_63

Newman Jr, W. J., Abell, S. K., Hubbard, P. D., McDonald, J., Otaala, J., \& Martini, M. (2004). Dilemmas of teaching inquiry in elementary science methods. Journal of Science teacher Education, 15(4), 257-279.

OCDE (2016). PISA: Resultados en foco. Recuperado desde: https://www.oecd.org/pisa/pisa-2015results-in-focus-ESP.pdf

Ogborn, J., Kress, G., y Martins, I. (1996). Explaining science in the classroom. London: McGrawHill Education.

Osborne, J. \& Patterson, A. (2011). Scientific argument and explanation: A necessary distinction? Science Education, 95(4), 627-638. Doi: 10.1002/sce.20438

Oktavianti, E., Handayanto, S. K., Wartono, W., \& Saniso, E. (2018). Students's scientific explanation in blended physics learning with e-scaffolding. Jurnal Pendidikan IPA Indonesia, 7(2), 181-186.

Park, J., Chang, J., Tang, K. S., Treagust, D. F. \& Won, M. (2020). Sequential patterns of students' drawing in constructing scientific explanations: focusing on the interplay among three levels of pictorial representation. International Journal of Science Education, 1-26.

Rappa, N. A., \& Tang, K. S. (2018). Integrating disciplinary-specific genre structure in discourse strategies to support disciplinary literacy. Linguistics and Education, 43, 1-12.

Ruiz-Primo, M. A., Li, M., Tsai, S.-P. \& Schneider, J. (2010). Testing one premise of scientific inquiry in science classrooms: Examining Students' scientific explanations and student learning. Journal of Research in Science Teaching, 47(5), 583-608. doi: 10.1002/tea.20356 
Sanmartí, N. (2007). Hablar, leer y escribir para aprender ciencia. En: P. Fernández. La competencia en comunicación lingüística en las áreas del currículo. Madrid, Colección Aulas de Verano: MEC.

Schwarz, C. V., Reiser, B. J., Davis, E. A., Kenyon, L., Achér, A., Fortus, D., ... \& Krajcik, J. (2009). Developing a learning progression for scientific modeling: Making scientific modeling accessible and meaningful for learners. Journal of Research in Science Teaching, 46(6), 632-654.

Songer, N. B., \& Gotwals, A. W. (2012). Guiding explanation construction by children at the entry points of learning progressions. Journal of Research in Science Teaching, 49(2), 141-165.

Sommer Lohrmann M. E., Cabello V. M. (2018) Supporting the Construction of Explanations of Natural Phenomena in Primary School Pupils. In: Andre T. (Ed) Advances in Human Factors in Training, Education, and Learning Sciences. AHFE 2017. Advances in Intelligent Systems and Computing, vol 596. Springer, Cham. Doi: 10.1007/978-3-319-60018-5_41

Tang, K. S. (2015). The PRO instructional strategy in the construction of scientific explanations. Teaching Science, 61(4), 14.

. (2016). Constructing scientific explanations through premise-reasoning-outcome (PRO): an exploratory study to scaffold students in structuring written explanations. International Journal of Science Education, 38(9), 1415-1440.

Thagard, P. (2012). The cognitive science of science: Explanation, discovery, and conceptual change. MIT Press.

van de Pol, J., Volman, M., \& Beishuizen, J. (2010). Scaffolding in teacher-student interaction: A decade of research. Educational Psychology Review, 22(3), 271-296. Doi: 10.1007/s10648-0109127-6

Vigotsky, L. S. (1979). El desarrollo de los procesos psicológicos superiores. Barcelona: Crítica.

Yao, J. X., \& Guo, Y. Y. (2018). Validity evidence for a learning progression of scientific explanation. Journal of Research in Science Teaching, 55(2), 299-317. Doi: 10.1002/tea.21420

Zangori, L. y Forbes, C. T. (2013). Preservice elementary teachers and explanation construction: knowledge-for-practice and knowledge-in-practice. Science Education, 97(2), 310-330. Doi: $10.1002 /$ sce. 21052

Zimmerman, C. (2000). The development of scientific reasoning skills. Developmental Review, 20(1), 99-149. Doi: 10.1006/drev.1999.0497 
